\title{
A Case Report of Triple-Positive Micropapillary Carcinoma of the Male Breast
}

\author{
Jamila Shuja $^{\mathrm{a}}$ Iftikhar Ahmad $^{\mathrm{b}}$ Sidra Arshad $^{\mathrm{c}}$ Hina Manzoor $^{\mathrm{a}}$ Shahwali Kakar $^{\mathrm{d}}$ \\ Khushnaseeb Ahmad ${ }^{a}$ \\ ${ }^{a}$ Center for Nuclear Medicine and Radiotherapy (CENAR), Quetta, Pakistan; \\ ${ }^{b}$ Institute of Radiotherapy and Nuclear Medicine (IRNUM), Peshawar, Pakistan; \\ ${ }^{c}$ Agha Khan University Hospital, Karachi, Pakistan; \\ ${ }^{\mathrm{d} B o l a n}$ Medical College, Quetta, Pakistan
}

\section{Established Facts}

- Micropapillary carcinoma (MPC) of the male breast is an exceedingly rare neoplasm.

\section{Novel Insights}

- We herein present a case of MPC of the male breast with triple-positive status. The patient responded promisingly to multimodality treatment and remained disease-free for over 2 years.

\section{Keywords}

Micropapillary carcinoma - Modified radical mastectomy · Lymphadenopathy · Triple-positive · Chemotherapy

\section{Summary}

Background: Micropapillary carcinoma (MPC), a morphologically distinct subtype of invasive ductal carcinoma, of the male breast is an exceedingly uncommon disease. Case Report: Herein, we report a case of triplepositive MPC of the male breast with axillary lymph node involvement and no recurrence for over 2 years. Specifically, a 60-year-old male patient presented with a hard, elastic, and well-defined painless mass in the right breast. The patient underwent unilateral (right) modified radical mastectomy with axillary clearance. Histopathology revealed MPC grade 3 and metastasis in 16/16 lymph nodes. Hormone receptor analysis demonstrated strong positivity (total score 08 ) for estrogen/progesterone receptors and overexpression (score $3+$ ) of human epider- mal growth factor receptor 2 . The patient received adjuvant chemotherapy ( 6 courses of CAF: cyclophosphamide, doxorubicin, and 5-fluorouracil), radiation, and tamoxifen. The patient has remained disease-free for over 2 years. Conclusion: This study demonstrates that triplepositive MPC of the male breast as a rare malignancy appears to respond promisingly to multimodality treatment.

(C) 2018 S. Karger GmbH, Freiburg

\section{Introduction}

Male breast cancer is a rare disease, accounting for less than $1 \%$ of all cancers affecting men [1]. Micropapillary carcinoma (MPC), a morphologically distinct and uncommon subtype of invasive ductal carcinoma, is an extremely scarce malignancy in males [2].

\section{KARGER}

() 2018 S. Karger GmbH, Freiburg 
Fig. 1. a, b Illustrative histopathology images of the resected micropapillary carcinoma. Hematoxylin and eosin staining $(40 \times)$ showing the lesion composed of nests and well-formed angular structures or morules of epithelial cells with surrounding retraction artifacts (empty spaces). Tumor cells have rounded-to-oval nuclei, finely granular chromatin, small nucleoli, and pale-to-clear scanty cytoplasm. The stroma is fibro-collagenous.
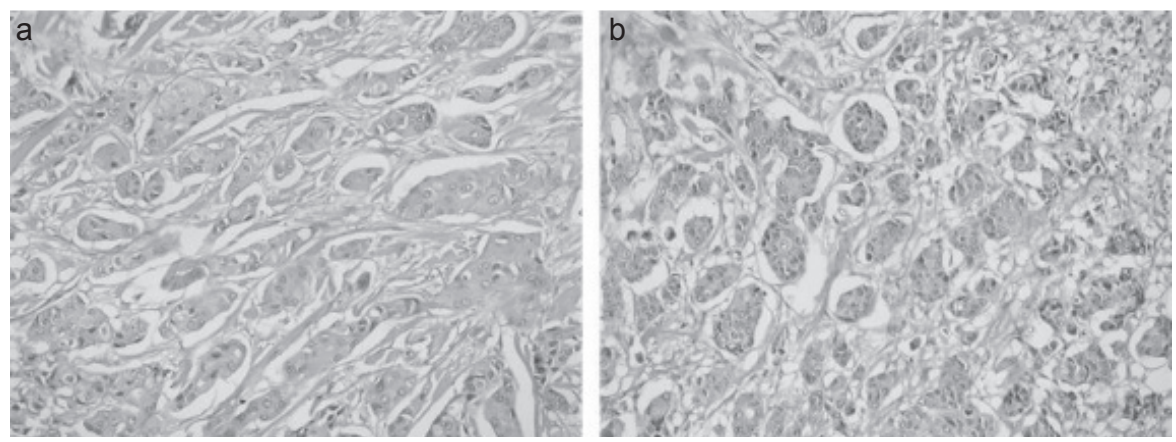

Clinically, MPC is typically associated with high manifestation of axillary lymph node (ALN) metastasis and local recurrence [3-5]. Although color Doppler ultrasound is considered as the most sensitive imaging tool for the assessment of papillary breast lesions [6], radiologic studies (including mammography) cannot accurately ascertain the diagnosis of MPC without histopathological evaluation $[7,8]$. The histopathology of MPC shows extensive angiolymphatic invasion and strong positivity for hormone receptors (i.e., estrogen/progesterone receptors (ER/PR)) and human epidermal growth factor receptor 2 (HER2) [9]. Multimodality treatment likely offers the best prognostic benefit in terms of disease-free and overall survival [10]. Specifically, it has been reported that the treatment strategy for MPC may comprise surgery (i.e., total mastectomy) in tandem with systemic chemotherapy and radiotherapy $[5,10]$. Moreover, hormone therapy has also been advocated for ER/PR-positive patients. Major prognostic factors for MPC include pathological stage at the time of diagnosis, ER/PR status, and HER2 overexpression $[4,5,9,11-13]$.

Here, we present a case of triple-positive MPC of the male breast managed with a multimodality treatment strategy. Specifically, the patient underwent modified radical mastectomy (MRM) with ALN clearance followed by chemo-, radio-, and hormone therapy. The patient is being followed up regularly and has remained disease-free for over 2 years.

\section{Case Report}

A 60-year-old male presented to us complaining of a painless mass in his right breast for some months. Physical examination of the patient illustrated a hard, elastic, and well-defined mass measuring $5 \times 4 \mathrm{~cm}^{2}$, adhering to both the superficial and the deep aponeurosis. No significant lymphadenopathy was present in the cervical, supraclavicular, and axillary regions. History of familial breast cancer, any other malignancy, or hormonal medication was negative; however, the clinical features were suspicious for malignancy.

The patient underwent unilateral (right) MRM with ALN dissection. Histopathology revealed poorly differentiated MPC (grade 3 ) according to the modified Bloom-Richardson grading system. The tumor size was $5 \times 4.5 \times 2.7$ $\mathrm{cm}^{3}$. A total of $16 \mathrm{ALN}$ were recovered and all tested positive for the presence of cancer cells; the size of largest lymph node was $2 \mathrm{~cm}$. Features included intraductal component present in a relative amount of $5 \%$ and comedo type with central necrosis. Illustrative histopathology images are shown in figure 1. Pathological TNM stage was evaluated as pT4, N3a, Mx. Hormone receptor analysis demonstrated a total intensity plus proportion score for each ER and PR of 08 , i.e., strongly positive. Moreover, HER2 was also overexpressed (score 3+).
Postoperatively, the patient underwent metastatic workup including X-ray chest, ultrasound abdomen and pelvis, and bone scintigraphy, which did not reveal further spread of the disease (i.e., M0). Consequently, the clinical stage of the disease was stage IIIC.

The therapeutic strategy comprised 6 courses of adjuvant chemotherapy (CAF: (cyclophosphamide $600 \mathrm{mg} / \mathrm{m}^{2}$, doxorubicin $60 \mathrm{mg} / \mathrm{m}^{2}$, and 5-fluorouracil $600 \mathrm{mg} / \mathrm{m}^{2}$, all on day 1 ), followed by external beam radiotherapy to the right chest wall and supraclavicular region (dose: $50 \mathrm{~Gy} / 25$ fractions). Afterwards, the patient received tamoxifen on the basis of positive status for ER/PR. Importantly, the patient refused trastuzumab due to financial constraints. Presently, the patient is on regular follow-up and has remained disease-free for over 2 years. Written and signed consent has been obtained from the patient for publishing this case report.

\section{Discussion}

This report presents the first case of MPC of the male breast from Pakistan, as part of our recently initiated project 'Centralized Registry Program for Uncommon Tumors' where incidences of rare tumors are being recorded [14-16]; this project aims to ascertain a reliable national database for uncommon tumors, enabling the assessment and comparison of rare tumors arising in Pakistan with similar studies. Moreover, the project/database will provide insight into treatment options for these uncommon tumors and their outcomes, with particular emphasis on local and individual circumstances and patient values.

Epidemiologic risk factors for male breast cancer have been previously described. Specifically, genetic factors (e.g., amplification of proto-oncogene c-Myc), gonadal disorders, obesity (i.e., hormonal dysfunction), alcohol intake, and radiation exposure are suspected to increase the risk of male breast cancer [17-22]. That said, we observed strong positivity of ER/PR and overexpression of HER2 in our patient and subsequently administered tamoxifen; trastuzumab was out of the patient's financial reach.

Established treatment protocols for MPC of the male breast are limited. Nevertheless, surgical intervention most likely offers some benefit towards a favorable prognosis. Importantly, MPC is typically associated with a high incidence of ALN metastases, necessitating extensive ALN dissection [10]. Our patient underwent MRM with ALN clearance, and all of the recovered 16 lymph nodes were positive. It is noteworthy that the number of nodes infiltrated by the tumor has been associated with disease prognosis, irrespective of the pathological type of breast cancer $[2,23]$. The postoperative treatment of the patient was planned on the basis of established protocols for invasive 
ductal carcinoma. Specifically, the patient was given 6 courses of adjuvant CAF chemotherapy followed by radiotherapy (50 Gy/25 fractions) to the chest wall and the supraclavicular regions.

Several studies have reported various treatment options and corresponding outcomes for male patients with MPC of the breast. For instance, a male patient treated with total mastectomy $(2 / 14$ positive ALN), adjuvant chemotherapy (paclitaxel followed by doxorubicin and cyclophosphamide), and radiotherapy developed liver metastases after 1 year and died after 2 years [1]. Another patient who presented with lung and adrenal metastases at the time of diagnosis and was treated with CEF-based chemotherapy (cyclophosphamide, epirubicin, and 5-fluorouracil) died 3 months after the initial diagnosis [24]. Another male patient with invasive MPC treated with mastectomy and tamoxifen (due to positive status for ER/PR) remained disease-free for 1 year [25]. In this study, the patient was disease-free for over 2 years after multimodality treatment (i.e., MRM followed by chemo-, radio-, and hormone therapy).

\section{Conclusion}

We presented an exceedingly uncommon case of stage IIIC, triple-positive MPC of the male breast managed with surgery and adjuvant chemoradiation. Specifically, unilateral MRM with ALN dissection (16/16 positive nodes) was followed by chemotherapy (CAF), radiotherapy (50 Gy), and hormonal therapy (tamoxifen). Systemic symptoms were relieved and the patient was disease-free for more than 2 years of follow-up. This study demonstrates that MPC of the male breast appears to respond promisingly to multimodality treatment. The literature review demonstrated a high likelihood of early ALN metastasis, contributing to the poor prognosis.

\section{Disclosure Statement}

The authors declare no conflict of interest. Further, the authors disclose no sponsorship or funding arrangements relating to this study.

\section{References}

1 El Hadj OEA, Chaar I, Goucha A, Belghith M, El May A, Gamoudi A: Pure micropapillary carcinoma of the male breast: report of a rare case. Breast Cancer Curr Res 2016;1:113.

2 Nassar H, Wallis T, Andea A, Dey J, Adsay V, Visscher D: Clinicopathologic analysis of invasive micropapillary differentiation in breast carcinoma. Mod Pathol 2001;14:836-841.

3 Joshi N, Pande C: Papillary carcinoma of the male breast diagnosed by fine needle aspiration cytology. Indian J Pathol Microbiol 1998;41:103-106.

4 Paterakos M, Watkin WG, Edgerton SM, Moore DH 2nd, Thor AD: Invasive micropapillary carcinoma of the breast: a prognostic study. Hum Pathol 1999;30: 1459-1463.

5 Chen AC, Paulino AC, Schwartz MR, et al: Populationbased comparison of prognostic factors in invasive micropapillary and invasive ductal carcinoma of the breast. Br J Cancer 2014;111:619-622.

6 Kim TH, Kang DK, Kim SY, Lee EJ, Jung YS, Yim H: Sonographic differentiation of benign and malignant papillary lesions of the breast. J Ultrasound Med 2008; 27:75-82.

7 Jones KN, Guimaraes LS, Reynolds CA, Ghosh K, Degnim AC, Glazebrook KN: Invasive micropapillary carcinoma of the breast: imaging features with clinical and pathologic correlation. Am J Roentgenol 2013;200: 689-695.

8 Lam WWM, Chu WCW, Tang APY, Tse G: Role of radiologic features in the management of papillary lesions of the breast. Am J Roentgenol 2006; 186:13221327.
Tang S, Yang J, Du Z, et al: Clinicopathologic study of invasive micropapillary carcinoma of the breast. Oncotarget 2017;8:42455-42465.

10 Taketani K, Tokunaga E, Yamashita N, et al: A case of invasive micropapillary carcinoma of the breast involving extensive lymph node metastasis. World J Surg Oncol 2014;12:84

11 Yu JI, Choi DH, Huh SJ, et al: Differences in prognostic factors and failure patterns between invasive micropapillary carcinoma and carcinoma with micropapillary component versus invasive ductal carcinoma of the breast: retrospective multicenter case-control study (KROG 13-06 ). Clin Breast Cancer 2015;15:353-361.

12 Yu JI, Choi DH, Park W, et al: Differences in prognostic factors and patterns of failure between invasive micropapillary carcinoma and invasive ductal carcinoma of the breast: matched case-control study. Breast 2010; 19:231-237.

13 Chen AC, Paulino AC, Schwartz MR, et al: Prognostic markers for invasive micropapillary carcinoma of the breast: a population-based analysis. Clin Breast Cancer 2013;13:133-139.

14 Sadiq M, Ahmad I, Shuja J, Ahmad K: Primary Ewing sarcoma of the kidney: a case report and treatment review. CEN Case Rep 2017;6:132-135.

15 Shuja J, Ahmad I, Ahmad K, et al: Pleuropulmonary blastoma. J Cancer Res Pract 2017;4:111-144.

16 Iftikhar S, Ahmad I, Qasmi IM, Ahmad K, Manzoor H: Tuberous sclerosis complex with sub-ependymal giant cell astrocytomas; a case report. J Cancer Res Pract 2017;4:147-150.
7 Trépant A, Hoorens A, Noël J: Pure invasive micropapillary carcinoma of the male breast: report of a rare case with C-MYC amplification. Pathol Res Pract 2014; 210:1164-1166.

18 Ron E, Ikeda T, Preston DL, Tokuoka S: Male breast cancer incidence among atomic bomb survivors. J Natl Cancer Inst 2005;97:603-605.

19 Burki T: Genomic profiling of male breast cancer. Lancet Oncol 2016;117:e141.

20 Brinton LA, Richesson DA, Gierach GL, et al: Prospective evaluation of risk factors for male breast cancer. J Natl Cancer Inst 2008;100:1477-1481.

21 Johansen Taber KA, Morisy LR, Osbahr AJ 3rd, Dickinson BD: Male breast cancer: risk factors, diagnosis, and management (review). Oncol Rep 2010;24:11151120

22 Bogarapu CB, Vayalapalli MR: Incidence of male breast cancer in a tertiary care hospital: a retrospective study with review of literature. Int J Contemp Med Res 2016;3:1803-1805.

23 McCready DR, Chapman JW, Hanna WM, et al: Factors affecting distant disease-free survival for primary invasive breast cancer: use of a log-normal survival model. Ann Surg Oncol 2000;7:416-426.

24 Erhan Y, Erhan Y, Zekioglu O: Pure invasive micropapillary carcinoma of the male breast: report of a rare case. Can J Surg 2005;48:156-157.

25 Tsushimi T, Mori H, Harada T, Ikeda Y, Ohnishi H: Invasive micropapillary carcinoma of the breast in a male patient: report of a case. Int J Surg Case Rep 2013; 4:988-991. 